\title{
Genotype $\times$ Environment Interaction and Stability analysis for Seed Yield and its Component Traits in Sunflower (Helianthus annuus L.)
}

\author{
B. Satish Chandra ${ }^{1 *}$, S. Sudheer Kumar ${ }^{2}$ and A.R.G. Ranganatha ${ }^{3}$
}

${ }^{1}$ Regional Agricultural Research Station, PJTSAU, Warangal-506007, Telangana, India

${ }^{2}$ Department of Genetics and Plant Breeding, PJTSAU, Rajendranagar, Hyderabad-30, Telangana, India

${ }^{3} I C A R$ - Indian Institute of Oilseeds Research, Hyderabad-30, Telangana, India

*Corresponding author

\section{A B S T R A C T}

Keywords

Stability, G $\times$ E

interaction, Seed yield,

Regression, Sunflower, Hybrids

Article Info

Accepted:

04 October 2018

Available Online:

10 November 2018
The Present investigation was carried out to evaluate 42 sunflower hybrids, their parents involving seven cytoplasmic male sterile lines and six restorer lines along with three checks for their stability across three different locations of Telangana during Rabi, 200809. Pooled analysis revealed that significant differences between genotypes and environments. Highly significant genotype $\times$ environment interaction for all the characters studied. The variance due to environment (linear) was highly significant for all the characters. The variance due to pooled deviations (non-linear) was significant for all the characters except head diameter revealing fluctuating performances of genotypes from linearity of response to varying environments. Based on stability performance three hybrids viz., CMS 17A $\times$ LTRR 341, ARM 243A × 3376R and ARM 238A × 3376R were identified as stable for seed yield and other traits, hence suitable for wider environments. Two hybrids, CMS 234A $\times$ RHA-6D-1R and CMS 852A $\times$ RHA-6D-1R were found suitable for favourable environments.

\section{Introduction}

Sunflower (Helianthus annuus L.) is one of the important oilseed crops after Groundnut, Rapeseed-Mustard and Soybean. Oilseeds form the second largest agricultural commodity after cereals in India accounting for 14 per cent of the country's gross cropped area and contribute for nearly five per cent of the gross national product and 10 per cent of the value of all agricultural products (Neelima et al., 2016). The presently cultivated varieties and hybrids though having high seed yield potential, they are erratic in their performance even under less varied conditions of cultivation. Apart from the biotic and abiotic stresses, lack of hybrids suitable to specific locations accounts for the decline in the area and productivity of sunflower. This warrants the attention of the plant breeders to evolve superior hybrids that would sustain well in the strainful situation. Therefore, assessment of its adaptability is of important concern. In breeding for wide adaptation, the aim is to obtain a variety, which performs well in nearly all environments (Cooper \& De-Lacy, 1994). 
Information on genotype $\times$ environment interaction leads to successful evaluation of stable genotype, which could be used for general cultivation. Yield is a complex quantitative character and is greatly influenced by environmental fluctuations; hence, the selection for superior genotypes based on yield per se at a single location in a year may not be very effective (Shrestha et al., 2012). Thus, evaluation of genotypes for stability of performance under varying environmental conditions for yield has become an essential part of any breeding program. A genotype is considered to be stable if its amongenvironment variance is small. This is called stability statistic, or a biological concept of stability. Productivity of a population is the function of its adaptation, whereas stability is the statistical measure of genotype $\times$ environment interaction (Kandil et al., 1990). $A$ significant $G \times E$ interaction for $a$ quantitative trait such as seed yield can seriously limit the efforts on selecting superior genotypes for improved cultivar development (Kang and Gorman, 1989). Testing of genotypes under different environmental situations differing in unpredictable variation is an accepted approach for selecting stable genotypes (Eberhart and Russel, 1966). Hence, the present study was undertaken to know the genotype-environment interaction and to identify stable and high yielding sunflower hybrids under changing environments.

\section{Materials and Methods}

The materials for the present study comprised 42 hybrids developed in a line $\times$ tester mating design involving seven CMS lines as females and six restorer lines as the male parents. The hybrids between seven female parents and six male parents were attempted during kharif, 2008 at research farm, Directorate of Oilseeds Research (Presently ICAR-Indian Institute of Oilseeds Research.), Hyderabad. The resulting
$42 \mathrm{~F}_{1}$ hybrids along with thirteen parents and three checks viz., KBSH-1, PAC-1091 and KBSH-44 were evaluated during rabi, 200809. Each entry was sown in a two rows of 4.5 $\mathrm{m}$ length with spacing of $60 \times 30 \mathrm{~cm}$ in a randomized block design replicated thrice at three different locations of Telangana viz., Directorate of Oilseeds Research, Hyderabad; Agricultural Research Station, Tandur for Southern Telangana agro-climatic zone and Regional Agricultural Research Station, Jagtial for Northern Telangana agro-climatic zone. All the recommended agronomic practices were followed to raise a healthy crop. The data were obtained on five random plants in each replication for eight quantitative characters i.e., days to maturity, plant height, head diameter, number of filled seeds per head, 100-seed weight, seed yield per plant, oil content and oil yield per plant. The mean values for all the traits across the environments were subjected to stability analysis as suggested by Eberhart and Russell (1966).

\section{Results and Discussions}

Pooled analysis of variance revealed that the genotypes and environments were significant for all the 8 characters (Table 1) indicating the diversity among the genotypes and environments studied. The GE interactions were significant for eight characters viz., days to maturity, plant height, head diameter, number of filled seeds per head, 100 seed weight, seed yield per plant, oil content and oil yield per plant. Significant genotype $\times$ environment interactions implies differential behaviour of genotypes under three different locations. Similar reports were earlier made by Laishram and Singh (1997), Prusti et al., (1999), Ahmed and Abdalla (2008). Partitioning of sum of squares into that of varieties, environments + (genotype $\times$ environment) and pooled error revealed that mean squares due to environments + 
(Genotype $\times$ Environment) were significant for all the eight characters reemphasizing the existence of variable response of genotypes to changing environments (GE interactions) for these traits. The findings are in accordance with Sankar Goud and Sarala (2004) and Balu et al., (2007). Sum of squares due to $E+(G \times$ E) was further partitioned into that of environment (linear), Genotype $\times$ Environment (linear) and pooled deviation. Significant variation due to environment (linear) was observed for all the eight characters studied revealing the linear contribution of environmental effects and additive environment variance on these characters. Similar results were reported by Sobhan Babu (2005). The mean sum of squares for pooled deviation was significant for all the eight characters, indicating the nonlinear response and unpredictable nature of the genotypes by significantly differing for stability. Significant non- linear responses were also observed earlier by Shankar Goud and Sarala (2004) and Balu et al., (2007), while both significant and non- significant linear responses were reported by Rukmini Devi et al., (2006) and Mahajan et al., (2009).

Environmental index reveals the favourability of an environment at a particular location. Breeze (1969) pointed out that the estimates of environmental index can provide the basis for identifying the favourable environments for the expression of maximum potential of the genotype. Based on positive values of environmental indices (Table 2), Hyderabad was found to be the most favourable location for number of filled seeds per head, seed yield per plant, head diameter, oil yield per plant and 100 seed weight. While Jagtial was the most favourable for days to maturity and oil content. The Tandur location was the most favourable for plant height. The results are in broad agreement with the results reported by Halaswamy et al., (2001). Since certain genotypes are searching for its favourable environment to express its fullest potential of yield and yield attributes. Hence an appropriate genotype has to be bred for each season.

Once it is known that genotypes interact with the environments significantly, the next task is to find out the most stable genotypes. The stability parameters .i.e., high mean performance $(\mu)$, regression coefficient $\left(b_{i}\right)$ and deviation from regression $\left(\mathrm{S}^{2} \mathrm{di}\right)$ were estimated for each genotype for each character separately. Both linear regression $\left(b_{i}\right)$ and deviation from regression $\left(\mathrm{S}^{2} \mathrm{~d}_{\mathrm{i}}\right)$ components of genotype $x$ environment interaction should be considered along with mean in judging the phenotypic stability of a genotype (Eberhart and Russell, 1966). Linear regression $\left(b_{i}\right)$ is a measure of response or sensitivity to environmental changes of a variety while deviation from regression measures the stability of genotypes with the lowest standard deviation $\left(S^{2} d_{i}=0\right)$ near to zero being the most stable and vice versa. Genotype with high mean performance, regression coefficient $\left(b_{i}\right.$ $=1$ ) approaching one and low deviation $\left(S^{2} \mathrm{di}\right)$ was considered to be an average stable genotype which would be expected to perform uniformly well over variable environments, if the regression coefficient $\left(b_{i}<1\right)$ being less than unity indicates a genotype to be above average stable response and which will be specially adapted to low yielding or unfavourable environments and if $b_{i}$ value $\left(b_{i}\right.$ $>1$ ) more than unity indicates a genotype with below average stable and such genotypes would perform better in high yielding or favourable environments, however its performance will be lower in stress environments compared to its genetic potentiality. Taking these parameters into consideration, the results obtained are discussed character wise.

For days to maturity, two parents CMS 852A, RES-834-1 and seven hybrids viz., CMS 234A 
$\times$ 3376R, CMS 852A × RES-834-1, CMS 17A $\times$ LTRR 341, ARM 243A × RHA-6D-1R, ARM 248A $\times$ RES-834-1, ARM 238A $\times$ LTRR 341 and ARM 248A $\times$ LTRR 341 were found early (Table 3) with unit regression coefficient (bi=0) values and non-significant deviation from regression $\left(\mathrm{S}^{2} \mathrm{di}\right)$ indicating that these genotypes are suitable for overall environmental conditions and they are considered as stable genotypes.

Similar findings were reported by Balu et al., (2007) and Mahajan et al., (2009). One hybrid ARM 238A $\times$ LTRR 341 is matured earlier than check KBSH-1 but recorded regression value is less than one (bi <1) and nonsignificantly deviating from regression, hence is adaptable to poor environments with more than average stability.

The stability parameters for plant height revealed that seven parents CMS 852A, CMS 89A, RES-834-1, 3376R, RHA-6D-1R, R 298 and $\mathrm{R} 649$ and 16 hybrids recorded regression coefficient (bi) value of one and nonsignificant deviation from regression, hence considered as widely adaptable to different environments with the average stability. These results were in accordance with Murali et al., (2004). Three hybrids viz., CMS 17A $\times$ LTRR 341, CMS 89A $\times$ RES-834-1 and ARM 243A $\times$ RHA-6D-1R recorded regression coefficient less than unity $(\mathrm{bi}<1)$ and considered as adaptable to poor environments. The hybrid CMS 852A $\times$ RES-834-1 exhibited more than one of regression coefficient (bi>1) values and considered to have less than average stability and are adaptable to favourable environments.

The stability parameters for head diameter revealed that parents viz., ARM 238A, CMS 17A, 3376R and one hybrid ARM 243A $\times$ RHA-6D-1R registered with unit regression coefficient (bi=0) values and non-significant deviation from linear regression, hence possess the average stability and is widely adaptable. These results were in accordance with the earlier findings of Rukmini Devi et al., (2006) and Balu et al., (2007).

For number of filled seeds per head (Table 4) based on unit regression $(\mathrm{bi}=0)$ and least deviation from regression all parents except 3376R and four hybrids viz., ARM 238A $\times$ 3376R, ARM 243A × 3376R, CMS 17A $\times$ $3376 \mathrm{R}$ and $\mathrm{ARM} 248 \mathrm{~A} \times 3376 \mathrm{R}$ were found stable genotypes, hence they are considered to be stable hybrids which can recommended for wider environments.

For 100 seed weight seven parents viz., CMS 234A, CMS 852A, CMS 89A, ARM 238A and ARM 248A, 3376R and R 298 recorded significant deviation from regression $\left(\mathrm{S}^{2} \mathrm{di}\right)$ values i.e., their performance cannot be predicted. None of the parents and hybrids was stable for all environments. However, one parent ARM 243A recorded higher mean value, regression coefficient value less than one $($ bi<1) and non-significant deviation from regression, indicating that this genotype was less sensitive to environmental changes and can be adapted only to poor environments hence is adaptable to poor environments with more than average stability. Among the hybrids, One hybrid ARM 248A × LTRR 341 recorded more than one of the regression coefficient (bi>1) value hence, is adaptable to favourable environments with less than the average stability. These results are in agreement with that of Shankar Goud and Sarala (2004) and Sobhan Babu (2005).

For Seed yield per plant, all breeding programmes invariably aim at achieving higher yields and hence, seed yield per plant is such an important character that decides the direction of the breeding programmes. Though yield is a complex character directly or indirectly influenced by yield component traits, the trait itself holds great importance and studied along with other characters. 
Table.1 ANOVA for yield and yield components for stability (Eberhart and Russell model) in sunflower

\begin{tabular}{|c|c|c|c|c|c|c|c|c|c|}
\hline \multicolumn{10}{|c|}{ Mean Sum of Squares } \\
\hline Source of variation & $\mathbf{d} \mathbf{f}$ & $\begin{array}{l}\text { Days to } \\
\text { maturity }\end{array}$ & $\begin{array}{l}\text { Plant } \\
\text { height }\end{array}$ & $\begin{array}{c}\text { Head } \\
\text { diameter }\end{array}$ & $\begin{array}{l}\text { No. of filled } \\
\text { seeds/head }\end{array}$ & $\begin{array}{c}\text { 100-seed } \\
\text { weight }\end{array}$ & $\begin{array}{l}\text { Seed yield } \\
\text { per plant }\end{array}$ & $\begin{array}{c}\text { Oil } \\
\text { content }\end{array}$ & $\begin{array}{l}\text { Oil yield } \\
\text { per plant }\end{array}$ \\
\hline Genotypes & 57 & $25.66 * *$ & $818.85^{* *}$ & $7.13 * *$ & $96603.02 * *$ & $1.57 * *$ & $235.96 * *$ & $11.36 * *$ & $34.46 * *$ \\
\hline$E+(G \times E)$ & 116 & $7.28 * *$ & $72.15 * *$ & $1.39 * *$ & $7378.82 * *$ & $0.29 * *$ & $12.58 * *$ & $3.36 * *$ & $1.76 * *$ \\
\hline Environments & 2 & $203.28 * *$ & $1083.27 * *$ & $53.22 * *$ & $67851.78 * *$ & $4.32 * *$ & $439.62 * *$ & $87.77 * *$ & $49.38 * *$ \\
\hline $\begin{array}{l}\text { Genotype } x \\
\text { Environments }\end{array}$ & 114 & $3.84 * *$ & $54.41 *$ & $0.48 * *$ & $6317.89 *$ & $0.22 *$ & $5.09 * *$ & $1.88^{*}$ & $0.92 * *$ \\
\hline Environment (Linear) & 1 & $406.56 * *$ & $2166.54 * *$ & $106.43 * *$ & $135703.56 * *$ & $8.65^{* *}$ & $879.23 * *$ & $175.54 * *$ & $98.76 * *$ \\
\hline $\begin{array}{l}\text { Genotype } x \\
\text { Environments (Linear) }\end{array}$ & 57 & $5.48 * *$ & $72.63 * *$ & $0.69 * *$ & $8406.35^{* *}$ & $0.29 * *$ & $7.34 * *$ & $2.49 * *$ & $1.31 * *$ \\
\hline $\begin{array}{l}\text { Pooled deviation (non- } \\
\text { linear) }\end{array}$ & 58 & $2.16^{* *}$ & $35.56 * *$ & 0.26 & $4156.50 * *$ & $0.14 * *$ & $2.80 * *$ & $1.25 * *$ & $0.52^{* * *}$ \\
\hline Pooled error & 171 & 0.40 & 6.27 & 0.21 & 737.41 & 0.004 & 1.05 & 0.59 & 0.19 \\
\hline
\end{tabular}

Table.2 Environmental indices for yield and yield components in sunflower

\begin{tabular}{|l|c|c|c|c|}
\hline \multirow{2}{*}{ Characters } & \multicolumn{3}{|c|}{ Locations } \\
\cline { 3 - 5 } & & Hyderabad & Jagtial & Tandur \\
\hline Days to maturity & $\mathrm{ij}$ & 1.701 & -2.006 & $\mathbf{0 . 3 0 5}$ \\
\hline Plant height & $\mathrm{ij}$ & -0.78 & 4.66 & $\mathbf{- 3 . 8 8}$ \\
\hline Head diameter & $\mathrm{ij}$ & 0.933 & 0.047 & $\mathbf{- 0 . 9 8 1}$ \\
\hline Number of filled seeds per head & $\mathrm{ij}$ & 42.081 & -24.807 & $\mathbf{- 1 7 . 2 7 3}$ \\
\hline 100-seed weight & $\mathrm{ij}$ & 0.18 & 0.14 & $\mathbf{- 0 . 3 1}$ \\
\hline Seed yield per plant & $\mathrm{ij}$ & 2.912 & -0.351 & $\mathbf{- 2 . 5 6 1}$ \\
\hline Oil content & $\mathrm{ij}$ & -1.145 & 1.300 & $\mathbf{- 0 . 1 5 5}$ \\
\hline Oil yield per plant & $\mathrm{ij}$ & $\mathbf{0 . 8 0 3}$ & $\mathbf{0 . 2 0 6}$ & $\mathbf{- 1 . 0 0 8}$ \\
\hline
\end{tabular}

Ij- Environmental index 
Table.3 Mean performance and stability parameters for days to maturity, plant height and head diameter in sunflower

\begin{tabular}{|c|c|c|c|c|c|c|c|c|c|}
\hline \multirow[t]{2}{*}{ PARENT/HYBRID } & \multicolumn{3}{|c|}{ Days to maturity } & \multicolumn{3}{|c|}{ plant height } & \multicolumn{3}{|c|}{ Head diameter } \\
\hline & Mean & bi & $\mathbf{S}^{2} \mathrm{di}$ & Mean & bi & $S^{2} d i$ & Mean & bi & $\mathrm{S}^{2} \mathrm{di}$ \\
\hline CMS-234A & 92.17 & 0.06 & $1.73 *$ & 125.92 & $1.88 *$ & -6.74 & 8.65 & 0.58 & -0.20 \\
\hline CMS-17A & 93.17 & 1.37 & $8.51 * *$ & 145.19 & 2.18 & -3.79 & 10.76 & 1.98 & 0.58 \\
\hline CMS-852A & 92.67 & 0.32 & 2.03 & 108.22 & 1.16 & 2.57 & 10.06 & 0.96 & -0.18 \\
\hline CMS-89A & 96.67 & 2.40 & -0.17 & 106.03 & 0.14 & 0.84 & 8.80 & 0.54 & 0.00 \\
\hline ARM-238A & 96.50 & $1.49 *$ & -0.41 & 150.83 & 3.10 & $20.51 *$ & 10.87 & 0.80 & -0.20 \\
\hline ARM-243A & 97.83 & 2.32 & -0.09 & 155.17 & 1.16 & 17.38 & 11.17 & 1.46 & $0.69 *$ \\
\hline ARM-248A & 99.00 & $1.75^{*}$ & -0.41 & 147.40 & -1.31 & -0.73 & 10.67 & 1.20 & -0.21 \\
\hline RES-834-1 & 93.67 & 1.41 & 0.84 & 107.27 & 0.43 & 8.33 & 9.74 & 1.22 & -0.19 \\
\hline LTRR-341 & 89.83 & 0.87 & $1.43 *$ & 118.47 & 3.35 & $212.84 * *$ & 7.43 & $-0.03 *$ & -0.20 \\
\hline 3376R & 94.33 & $0.41 *$ & -0.41 & 106.15 & 0.07 & -2.01 & 10.27 & 0.85 & -0.11 \\
\hline RHA-6D-1R & 94.50 & 0.53 & -0.37 & 131.77 & 1.87 & -6.16 & 8.00 & 0.33 & 0.01 \\
\hline R-298 & 94.33 & 0.27 & $6.23 * *$ & 119.33 & -0.41 & 17.21 & 7.90 & 0.63 & -0.17 \\
\hline R-649 & 95.50 & 1.92 & -0.15 & 124.10 & 0.29 & -5.97 & 8.90 & 0.50 & -0.19 \\
\hline CMS-234A $\times$ RES-834-1 & 93.00 & 1.59 & -0.06 & 148.55 & -0.58 & $248.86 * *$ & 10.65 & 1.34 & -0.16 \\
\hline CMS-234A $\times$ LTRR-341 & 92.83 & -0.02 & -0.25 & 150.33 & -1.50 & $75.20 * *$ & 10.28 & 0.63 & 0.18 \\
\hline CMS-234A $\times 3376 R$ & 89.33 & -0.45 & 0.33 & 153.17 & 1.31 & $37.53 *$ & 12.57 & 1.89 & $0.91 *$ \\
\hline CMS-234A $\times$ RHA-6D-1R & 93.00 & 0.76 & $11.01 * *$ & 155.43 & -0.09 & $54.35 * *$ & 12.29 & 1.22 & -0.12 \\
\hline CMS-234A $\times$ R-298 & 93.50 & -0.10 & 0.02 & 155.17 & -1.00 & $456.05 * *$ & 13.51 & 2.30 & $1.65 *$ \\
\hline CMS-234A $\times$ R-649 & 95.50 & 1.12 & 0.25 & 123.76 & -0.52 & -4.49 & 10.73 & 0.78 & -0.17 \\
\hline CMS-17A $\times$ RES-834-1 & 100.17 & 1.28 & $1.29 *$ & 143.29 & 2.08 & -5.86 & 11.82 & 0.44 & -0.18 \\
\hline CMS-17A $\times$ LTRR-341 & 89.83 & 0.34 & 0.93 & 140.25 & $-0.12 *$ & -6.62 & 11.33 & 1.66 & -0.06 \\
\hline CMS-17A $\times 3376 R$ & 97.67 & 1.87 & -0.33 & 146.55 & 1.34 & $52.38 * *$ & 11.83 & 1.62 & 0.40 \\
\hline CMS-17A $\times$ RHA-6D-1R & 95.00 & 0.63 & 0.32 & 155.97 & 1.01 & $155.69 * *$ & 13.68 & $1.60 *$ & -0.21 \\
\hline CMS-17A $\times$ R-298 & 98.00 & $2.71 *$ & -0.37 & 143.98 & 0.58 & 5.61 & 11.53 & 1.27 & -0.03 \\
\hline CMS-17A $\times$ R-649 & 100.00 & $-0.79 *$ & -0.32 & 132.18 & 1.96 & $60.69 * *$ & 10.92 & $-0.92 *$ & -0.20 \\
\hline CMS-852A $\times$ RES-834-1 & 89.50 & 0.19 & 0.51 & 130.65 & $1.54 *$ & -6.72 & 9.78 & $1.15^{*}$ & -0.21 \\
\hline CMS-852A $\times$ LTRR-341 & 92.50 & 0.56 & $3.86 * *$ & 137.80 & 0.76 & -5.53 & 10.50 & 1.02 & 0.24 \\
\hline CMS-852A $\times 3376 R$ & 100.17 & 2.50 & 0.91 & 151.82 & 2.18 & -5.43 & 10.95 & 0.16 & -0.16 \\
\hline CMS-852A $\times$ RHA-6D-1R & 96.67 & 2.04 & -0.34 & 143.83 & 1.76 & 18.57 & 13.43 & 0.54 & -0.07 \\
\hline CMS-852A $\times$ R-298 & 98.50 & 2.12 & 0.22 & 127.12 & 0.93 & -4.85 & 10.43 & 0.22 & -0.02 \\
\hline
\end{tabular}


Contd...

\begin{tabular}{|c|c|c|c|c|c|c|c|c|c|}
\hline \multirow[t]{2}{*}{ PARENT/HYBRID } & \multicolumn{3}{|c|}{ Days to maturity } & \multicolumn{3}{|c|}{ plant height } & \multicolumn{3}{|c|}{ Head diameter } \\
\hline & Mean & bi & $\mathbf{S}^{2} \mathbf{d i}$ & Mean & bi & $S^{2}$ di & Mean & bi & $S^{2} \mathbf{d i}$ \\
\hline CMS-852A × R-649 & 96.67 & 0.78 & $3.48 * *$ & 139.25 & 0.17 & -1.31 & 10.88 & 0.90 & 0.06 \\
\hline CMS-89A $\times$ RES-834-1 & 98.50 & 1.32 & -0.17 & 140.38 & $-0.85^{*}$ & -6.75 & 11.33 & 1.92 & -0.05 \\
\hline CMS-89A $\times$ LTRR-341 & 97.33 & 2.25 & $18.12 * *$ & 122.63 & -0.15 & -0.85 & 9.47 & -0.09 & 0.04 \\
\hline CMS-89A $\times$ 3376R & 98.17 & 2.73 & -0.03 & 125.73 & 0.98 & -6.32 & 10.87 & 0.58 & -0.15 \\
\hline CMS-89A $\times$ RHA-6D-1R & 96.00 & 1.19 & $3.69 * *$ & 144.50 & 1.17 & $38.37^{*}$ & 11.25 & 0.95 & $1.02 *$ \\
\hline CMS-89A $\times$ R-298 & 91.17 & -0.01 & $4.75 * *$ & 137.35 & 0.83 & 2.10 & 11.63 & $1.51 *$ & -0.21 \\
\hline CMS-89A $\times$ R-649 & 95.17 & 1.37 & $8.51 * *$ & 123.72 & 0.49 & 15.83 & 11.77 & 1.67 & 0.17 \\
\hline ARM-238A $\times$ RES-834-1 & 96.67 & 0.65 & -0.21 & 149.27 & 3.70 & $219.31 * *$ & 11.70 & 1.01 & -0.02 \\
\hline ARM-238A × LTRR-341 & 91.83 & $0.67 *$ & -0.41 & 145.47 & 1.27 & -3.77 & 12.85 & 0.03 & 0.33 \\
\hline ARM-238A $\times 3376 R$ & 96.33 & 1.27 & 0.52 & 154.52 & 2.16 & $152.41 * *$ & 12.37 & 1.26 & $0.81^{*}$ \\
\hline ARM-238A $\times$ RHA-6D-1R & 91.67 & -0.47 & 1.19 & 167.67 & 2.17 & $36.08^{*}$ & 12.33 & 0.49 & -0.19 \\
\hline ARM-238A $\times$ R-298 & 96.83 & 1.76 & $4.94 * *$ & 165.17 & 2.12 & $316.24 * *$ & 11.77 & 1.61 & -0.07 \\
\hline ARM-238A $\times$ R-649 & 99.33 & 1.79 & 0.17 & 159.07 & 0.91 & $219.96^{* *}$ & 12.80 & 1.48 & 0.07 \\
\hline ARM-243A $\times$ RES-834-1 & 92.33 & 1.04 & 0.23 & 143.07 & 1.30 & -6.44 & 11.55 & 1.46 & 0.14 \\
\hline ARM-243A x LTRR-341 & 94.50 & 1.22 & -0.39 & 149.83 & 1.92 & $95.76^{* *}$ & 12.58 & $1.57 *$ & -0.21 \\
\hline ARM-243A $\times 3376 R$ & 94.67 & 1.54 & $1.56^{*}$ & 155.60 & 2.16 & $48.43 * *$ & 12.68 & 1.25 & 0.44 \\
\hline ARM-243A $\times$ RHA-6D-1R & 91.17 & 0.39 & -0.29 & 153.68 & $0.24^{*}$ & -6.66 & 13.83 & 1.67 & 0.45 \\
\hline ARM-243A $\times$ R-298 & 91.17 & -0.21 & $1.45^{*}$ & 149.92 & -1.15 & 3.01 & 13.35 & 0.97 & -0.20 \\
\hline ARM-243A $\times$ R-649 & 94.17 & 0.85 & $2.70 *$ & 144.46 & 0.87 & $32.84 *$ & 13.15 & $1.02 *$ & -0.21 \\
\hline ARM-248A $\times$ RES-834-1 & 91.17 & 0.91 & -0.11 & 162.65 & 1.38 & $20.67 *$ & 11.65 & 1.03 & $0.88 *$ \\
\hline ARM-248A $\times$ LTRR-341 & 91.83 & 0.31 & 0.09 & 170.28 & 0.88 & $147.14 * *$ & 12.47 & 0.48 & -0.04 \\
\hline ARM-248A $\times 3376 R$ & 95.17 & 1.61 & -0.38 & 148.82 & 1.36 & -6.65 & 12.98 & 0.39 & 0.24 \\
\hline ARM-248A $\times$ RHA-6D-1R & 96.17 & 0.65 & $19.82 * *$ & 157.05 & 0.64 & $63.38 * *$ & 12.65 & 0.65 & -0.20 \\
\hline ARM-248A $\times$ R-298 & 94.83 & 0.11 & $3.67 * *$ & 169.37 & 1.15 & $20.82 *$ & 11.85 & 0.95 & -0.02 \\
\hline ARM-248A $\times$ R-649 & 92.00 & 0.76 & $11.01 * *$ & 139.72 & 0.38 & $64.80 * *$ & 13.18 & 2.03 & -0.13 \\
\hline KBSH 1 & 93.67 & 0.45 & 0.33 & 152.85 & 1.89 & 19.43 & 13.78 & 1.18 & -0.21 \\
\hline PAC 1091 & 95.17 & 0.29 & -0.32 & 140.11 & 1.51 & $86.67 * *$ & 12.24 & 1.06 & -0.21 \\
\hline KBSH 44 & 99.67 & 1.31 & $9.67 * *$ & 159.18 & 2.92 & $56.14 * *$ & 13.22 & 1.04 & -0.21 \\
\hline SE of bi & & 0.62 & & & 1.3 & & & 0.39 & \\
\hline
\end{tabular}

*: Significant at 5\% level; **: Significant at $1 \%$ level; 
Table.4 Mean performance and stability parameters for Number of filled seeds per head, 100 seed weight and Seed yield per plant in sunflower

\begin{tabular}{|c|c|c|c|c|c|c|c|c|c|}
\hline \multirow[t]{2}{*}{ PARENT/HYBRID } & \multicolumn{3}{|c|}{ Number of filled seeds per head } & \multicolumn{3}{|c|}{100 seed weight } & \multicolumn{3}{|c|}{ Seed yield per plant } \\
\hline & Mean & bi & $S^{2}$ di & Mean & bi & $S^{2}$ di & Mean & bi & $\mathbf{S}^{2} \mathrm{di}$ \\
\hline CMS-234A & 337.20 & 0.61 & -756.62 & 3.51 & 0.88 & $0.04 * *$ & 11.21 & 0.55 & -0.49 \\
\hline CMS-17A & 445.33 & 1.58 & -379.98 & 3.96 & $2.35^{*}$ & -0.003 & 17.35 & 1.51 & -0.68 \\
\hline CMS-852A & 341.92 & 0.29 & -651.41 & 4.97 & 1.02 & $0.06^{* *}$ & 16.15 & $0.69 *$ & -1.10 \\
\hline CMS-89A & 318.60 & 0.34 & 1388.42 & 2.89 & 1.84 & $0.04 * *$ & 8.47 & 0.29 & -1.06 \\
\hline ARM-238A & 465.90 & 1.91 & 865.51 & 3.60 & 0.58 & $0.10 * *$ & 15.91 & 0.96 & 0.37 \\
\hline ARM-243A & 346.64 & 1.05 & -751.92 & 6.07 & $0.49 *$ & -0.003 & 20.02 & 0.99 & -0.30 \\
\hline ARM-248A & 402.27 & 0.72 & 228.52 & 4.73 & 1.35 & $0.04 * *$ & 18.18 & 0.53 & -1.08 \\
\hline RES-834-1 & 418.20 & 1.57 & -588.87 & 3.58 & 0.31 & 0.06 & 14.49 & 0.87 & 4.70* \\
\hline LTRR-341 & 310.39 & 0.22 & -190.91 & 3.38 & $0.56^{*}$ & -0.003 & 9.76 & 0.03 & -0.82 \\
\hline 3376R & 412.91 & 0.47 & $6534.00 * *$ & 3.02 & 1.93 & $0.08 * *$ & 11.67 & 0.39 & -0.99 \\
\hline RHA-6D-1R & 316.29 & 0.46 & 490.60 & 2.81 & 0.41 & -0.004 & 8.33 & 0.41 & -0.79 \\
\hline R-298 & 400.56 & 2.34 & 2233.82 & 2.80 & -0.08 & $0.32 * *$ & 10.51 & 0.55 & 1.47 \\
\hline R-649 & 575.16 & 1.93 & 165.88 & 2.66 & $-0.26 *$ & -0.004 & 14.78 & 0.52 & -0.60 \\
\hline CMS-234A $\times$ RES-834-1 & 527.16 & 1.31 & 139.59 & 4.80 & 0.72 & $0.04 * *$ & 24.32 & 1.01 & $8.19 * *$ \\
\hline CMS-234A $\times$ LTRR-341 & 488.99 & -0.19 & -67.24 & 4.41 & 0.99 & $0.14 * *$ & 20.73 & 0.40 & 0.17 \\
\hline CMS-234A $\times 3376 R$ & 595.80 & 1.42 & -287.48 & 5.08 & -0.45 & $0.01 *$ & 29.25 & 0.82 & $3.33 *$ \\
\hline CMS-234A $\times$ RHA-6D-1R & 889.29 & 1.40 & $8398.35 * *$ & 4.30 & 0.45 & $0.12 * *$ & 37.63 & $1.96^{*}$ & -1.04 \\
\hline CMS-234A $\times$ R-298 & 753.08 & -0.45 & $5765.15^{* *}$ & 4.10 & 2.02 & $0.13 * *$ & 29.89 & 1.05 & 0.81 \\
\hline CMS-234A $\times$ R-649 & 555.74 & 0.60 & 595.66 & 3.54 & 0.34 & -0.003 & 19.02 & 0.56 & -0.51 \\
\hline CMS-17A $\times$ RES-834-1 & 766.86 & -1.22 & $6262.48 * *$ & 4.34 & 0.54 & $0.10 * *$ & 32.36 & 0.50 & $7.76 * *$ \\
\hline CMS-17A $\times$ LTRR-341 & 862.66 & 2.03 & $5579.12 * *$ & 4.51 & 0.11 & $0.08 * *$ & 38.06 & 2.02 & 0.78 \\
\hline CMS-17A $\times 3376 R$ & 812.51 & 4.18 & -593.86 & 4.35 & 0.50 & $0.43 * *$ & 33.39 & 1.66 & -0.61 \\
\hline CMS-17A $\times$ RHA-6D-1R & 871.86 & -1.42 & $9508.79 * *$ & 5.11 & 3.50 & $0.08 * *$ & 42.94 & 1.41 & $3.95 *$ \\
\hline CMS-17A $\times$ R-298 & 653.69 & -0.91 & $13761.79 * *$ & 4.37 & 2.76 & $0.44 * *$ & 27.38 & 1.00 & $8.39 * *$ \\
\hline CMS-17A $\times$ R-649 & 612.60 & -0.84 & $12727.25 * *$ & 3.16 & 1.81 & $0.07 * *$ & 18.42 & -0.01 & 0.04 \\
\hline CMS-852A $\times$ RES-834-1 & 660.29 & -0.91 & $8246.77 * *$ & 3.36 & 0.51 & $0.03 * *$ & 21.50 & -0.32 & $3.91 *$ \\
\hline CMS-852A $\times$ LTRR-341 & 716.20 & 1.41 & 1604.51 & 3.99 & 0.63 & $0.18 * *$ & 27.87 & 1.71 & 0.72 \\
\hline CMS-852A $\times 3376 R$ & 657.09 & 2.21 & -723.81 & 3.76 & 0.94 & 0.01 & 24.04 & 1.41 & -1.02 \\
\hline CMS-852A $\times$ RHA-6D-1R & 861.44 & 0.85 & $4214.22 *$ & 4.41 & 0.84 & $0.16 * *$ & 37.24 & $1.91 *$ & -1.08 \\
\hline CMS-852A $\times$ R-298 & 634.39 & -0.06 & -645.19 & 3.56 & 1.31 & $0.12 * *$ & 21.77 & 0.87 & -1.02 \\
\hline
\end{tabular}


Contd...

\begin{tabular}{|c|c|c|c|c|c|c|c|c|c|}
\hline \multirow[t]{2}{*}{ PARENT/HYBRID } & \multicolumn{3}{|c|}{ Number of filled seeds per head } & \multicolumn{3}{|c|}{100 seed weight } & \multicolumn{3}{|c|}{ Seed yield per plant } \\
\hline & Mean & bi & $S^{2} \mathrm{di}$ & Mean & bi & $S^{2} \mathbf{d i}$ & Mean & bi & $\mathbf{S}^{2} \mathbf{d i}$ \\
\hline CMS-89A $\times$ RES-834-1 & 748.73 & 1.19 & $64073.09 * *$ & 5.03 & 5.36 & $0.59 * *$ & 34.84 & 2.15 & -0.55 \\
\hline CMS-89A $\times$ 3376R & 626.87 & 0.59 & $2392.25^{*}$ & 3.10 & 0.45 & $0.05 * *$ & 18.82 & 0.82 & -0.84 \\
\hline CMS-89A $\times$ RHA-6D-1R & 839.12 & -3.26 & $34974.23 * *$ & 4.42 & 0.39 & $1.88 * *$ & 35.33 & 1.04 & -0.29 \\
\hline CMS-89A $\times$ R-298 & 542.78 & 4.20 & $10773.04 * *$ & 3.37 & -2.00 & $0.51 * *$ & 16.69 & 0.58 & 1.10 \\
\hline ARM-238A $\times$ LTRR-341 & 753.13 & -0.98 & $5085.68 * *$ & 3.55 & $-0.09 *$ & -0.003 & 26.15 & -0.79 & 0.78 \\
\hline ARM-238A $\times 3376 R$ & 936.12 & 1.31 & 868.46 & 4.00 & 1.69 & $0.02 *$ & 36.87 & 2.26 & 2.30 \\
\hline ARM-238A $\times$ RHA-6D-1R & 556.24 & -0.50 & $7563.80 * *$ & 4.37 & 1.11 & $0.89 * *$ & 23.42 & 1.44 & -0.70 \\
\hline ARM-238A $\times$ R-298 & 734.92 & 1.79 & $11917.10 * *$ & 4.06 & 1.11 & -0.003 & 28.98 & 0.92 & $16.79 * *$ \\
\hline ARM-238A $\times$ R-649 & 521.29 & 0.27 & $9199.05 * *$ & 4.79 & 2.84 & $0.32 * *$ & 23.76 & 1.19 & $12.39 * *$ \\
\hline ARM-243A $\times$ RES-834-1 & 722.62 & 2.07 & $5343.57 * *$ & 4.18 & 1.15 & -0.003 & 29.38 & 2.07 & 2.28 \\
\hline ARM-243A $\times$ R-649 & 534.55 & 2.44 & -470.64 & 4.79 & 1.77 & $0.17 * *$ & 24.67 & 1.74 & -0.97 \\
\hline ARM-248A $\times$ RES-834-1 & 599.53 & 2.24 & $2532.55^{*}$ & 4.36 & 0.62 & $1.11 * *$ & 25.10 & 0.74 & $29.24 * *$ \\
\hline ARM-248A $\times$ LTRR-341 & 574.09 & 1.06 & -448.57 & 4.91 & $2.00 *$ & -0.003 & 27.12 & 1.60 & 1.53 \\
\hline ARM-248A $\times 3376 R$ & 790.53 & -0.16 & 1544.87 & 3.97 & 0.53 & $0.27 * *$ & 30.48 & -0.54 & $3.68 *$ \\
\hline ARM-248A $\times$ RHA-6D-1R & 809.52 & 4.32 & $11823.54 * *$ & 4.64 & 0.02 & $0.31 * *$ & 36.39 & 2.25 & $9.00 *$ \\
\hline ARM-248A $\times$ R-298 & 576.10 & 3.07 & $2524.13^{*}$ & 4.93 & 0.52 & $1.12 * *$ & 27.02 & 1.32 & $14.76^{* *}$ \\
\hline ARM-248A $\times$ R-649 & 634.32 & 2.40 & $3470.87 *$ & 4.82 & 1.43 & $0.52 * *$ & 29.27 & 1.08 & -0.88 \\
\hline KBSH 1 & 719.90 & 0.08 & -738.35 & 4.34 & 1.40 & 0.00 & 31.13 & $0.40 *$ & -1.09 \\
\hline PAC 1091 & 820.53 & -0.63 & $3429.38 *$ & 4.32 & 2.00 & $0.11 * *$ & 34.45 & 1.00 & -1.02 \\
\hline KBSH 44 & 754.31 & -0.28 & -662.78 & 5.00 & 1.77 & $0.03 *$ & 36.65 & 1.03 & 0.14 \\
\hline SE of bi & & 1.50 & & & 1.22 & & & 0.46 & \\
\hline
\end{tabular}

$*$ : Significant at $5 \%$ level; **: Significant at $1 \%$ level 
Table.5 Mean performance and stability parameters for oil content and oil yield per plant in sunflower

\begin{tabular}{|c|c|c|c|c|c|c|}
\hline \multirow[t]{2}{*}{ PARENT/HYBRID } & \multicolumn{3}{|c|}{ Oil content } & \multicolumn{3}{|c|}{ Oil yield per plant } \\
\hline & Mean & bi & $S^{2}$ di & Mean & bi & $\mathbf{S}^{2} \mathrm{di}$ \\
\hline CMS-234A & 34.25 & -0.17 & 0.30 & 3.84 & 0.40 & 0.08 \\
\hline CMS-17A & 34.53 & 1.16 & $8.06 * *$ & 5.93 & 1.09 & -0.19 \\
\hline CMS-852A & 39.37 & 0.68 & -0.56 & 6.35 & 0.72 & -0.19 \\
\hline CMS-89A & 37.54 & 2.26 & -0.16 & 3.17 & 0.22 & -0.12 \\
\hline ARM-238A & 35.87 & 1.41 & $4.79 * *$ & 5.73 & 1.24 & 0.01 \\
\hline ARM-243A & 38.59 & $2.07 *$ & -0.57 & 7.70 & 0.83 & -0.20 \\
\hline ARM-248A & 37.42 & 0.71 & -0.50 & 6.80 & 0.48 & -0.20 \\
\hline RES-834-1 & 37.68 & 0.36 & $2.87 *$ & 5.44 & 0.50 & $1.23 * *$ \\
\hline LTRR-341 & 32.75 & 0.75 & -0.55 & 3.20 & $-0.04 *$ & -0.20 \\
\hline 3376R & 36.45 & 0.69 & 0.73 & 4.25 & 0.45 & -0.19 \\
\hline RHA-6D-1R & 40.77 & 0.74 & 1.61 & 3.39 & 0.41 & -0.19 \\
\hline R-298 & 37.70 & 1.00 & $2.63 *$ & 3.95 & 0.57 & 0.05 \\
\hline R-649 & 34.76 & 1.53 & 1.42 & 5.13 & $0.53 *$ & -0.20 \\
\hline CMS-234A $\times$ RES-834-1 & 38.19 & 1.14 & 0.11 & 9.25 & 0.56 & $1.10 *$ \\
\hline CMS-234A $\times$ LTRR-341 & 34.93 & 0.69 & -0.37 & 7.24 & 0.19 & -0.10 \\
\hline CMS-234A $\times 3376 R$ & 37.71 & 1.46 & -0.56 & 11.01 & 0.58 & -0.05 \\
\hline CMS-234A $\times$ RHA-6D-1R & 38.00 & 0.76 & $10.09 * *$ & 14.36 & $2.91 *$ & -0.19 \\
\hline CMS-234A $\times$ R-298 & 36.42 & $2.01 *$ & -0.58 & 10.86 & 0.78 & -0.19 \\
\hline CMS-234A $\times$ R-649 & 38.30 & 1.37 & -0.58 & 7.28 & 0.61 & 0.04 \\
\hline CMS-17A $\times$ RES-834-1 & 35.22 & 0.96 & 0.38 & 11.42 & 0.89 & $1.82 * *$ \\
\hline CMS-17A $\times$ LTRR-341 & 33.19 & -0.39 & -0.24 & 12.67 & 2.03 & $1.89 * *$ \\
\hline CMS-17A $\times 3376 R$ & 34.54 & 1.41 & $3.34 *$ & 11.54 & $1.87^{*}$ & -0.20 \\
\hline CMS-17A $\times$ RHA-6D-1R & 36.12 & 1.10 & 0.47 & 15.52 & 1.79 & $1.01 *$ \\
\hline CMS-17A $\times$ R-298 & 36.14 & -0.34 & -0.13 & 9.90 & 0.66 & $2.57 * *$ \\
\hline CMS-17A $\times$ R-649 & 35.08 & 0.65 & $7.30 * *$ & 6.47 & 0.43 & 0.27 \\
\hline CMS-852A $\times$ RES-834-1 & 38.09 & 1.93 & 1.44 & 8.19 & $-0.91 *$ & -0.20 \\
\hline CMS-852A $\times$ LTRR-341 & 36.88 & -0.51 & 1.50 & 10.32 & 2.03 & $1.80 * *$ \\
\hline CMS-852A $\times 3376 R$ & 36.70 & 2.13 & 0.27 & 8.76 & 1.08 & -0.17 \\
\hline CMS-852A $\times$ RHA-6D-1R & 39.23 & 0.84 & -0.56 & 14.59 & 1.99 & -0.13 \\
\hline CMS-852A $\times$ R-298 & 36.90 & $0.38 *$ & -0.59 & 8.03 & 0.85 & -0.09 \\
\hline
\end{tabular}




\begin{tabular}{|c|c|c|c|c|c|c|}
\hline \multirow[t]{2}{*}{ PARENT/HYBRID } & \multicolumn{3}{|c|}{ Oil content } & \multicolumn{3}{|c|}{ Oil yield per plant } \\
\hline & Mean & bi & $S^{2}$ di & Mean & bi & $\mathrm{S}^{2} \mathrm{di}$ \\
\hline CMS-852A $\times$ R-649 & 40.22 & 1.77 & 0.37 & 10.39 & 1.43 & -0.03 \\
\hline CMS-89A $\times$ RES-834-1 & 40.53 & 1.35 & 1.44 & 14.04 & 1.86 & 0.06 \\
\hline CMS-89A $\times$ LTRR-341 & 38.99 & 0.06 & -0.44 & 5.59 & 0.77 & -0.15 \\
\hline CMS-89A $\times$ 3376R & 37.41 & 1.45 & -0.53 & 7.02 & 0.85 & -0.10 \\
\hline CMS-89A $\times$ RHA-6D-1R & 38.64 & 1.04 & -0.37 & 13.66 & 1.21 & 0.09 \\
\hline CMS-89A $\times$ R-298 & 40.09 & 1.66 & $3.58 * *$ & 6.68 & 0.47 & 0.33 \\
\hline CMS-89A $\times$ R-649 & 38.63 & 2.33 & $11.46^{* *}$ & 9.15 & 0.61 & -0.05 \\
\hline ARM-238A $\times$ RES-834-1 & 39.85 & 0.65 & -0.07 & 14.41 & $1.44^{*}$ & -0.20 \\
\hline ARM-238A $\times$ LTRR-341 & 35.44 & $-0.38 *$ & -0.57 & 9.26 & -0.85 & -0.12 \\
\hline ARM-238A $\times 3376 R$ & 36.72 & 1.22 & $7.83 * *$ & 13.60 & 3.10 & 0.23 \\
\hline ARM-238A $\times$ RHA-6D-1R & 39.29 & 1.37 & $4.25^{*}$ & 9.22 & 1.91 & -0.14 \\
\hline ARM-238A $\times$ R-298 & 40.18 & 1.20 & 0.48 & 11.60 & 0.29 & $2.13 * *$ \\
\hline ARM-238A × R-649 & 38.86 & 1.99 & 1.30 & 9.19 & 1.05 & $0.99 *$ \\
\hline ARM-243A $\times$ RES-834-1 & 39.98 & 1.38 & -0.39 & 11.72 & 2.43 & 0.04 \\
\hline ARM-243A $\times$ LTRR-341 & 36.61 & 0.43 & $3.53 * *$ & 8.78 & $1.66^{*}$ & -0.20 \\
\hline ARM-243A $\times 3376 R$ & 37.78 & 1.62 & $2.11^{*}$ & 13.95 & 1.48 & 0.50 \\
\hline ARM-243A $\times$ RHA-6D-1R & 39.62 & 1.81 & $1.88 *$ & 13.44 & -0.50 & 0.08 \\
\hline ARM-243A $\times$ R-298 & 39.49 & 0.79 & 0.36 & 14.02 & 1.47 & 0.09 \\
\hline ARM-243A $\times$ R-649 & 39.29 & 1.30 & $1.79 *$ & 9.70 & 2.04 & -0.09 \\
\hline ARM-248A $\times$ RES-834-1 & 39.29 & 0.58 & 0.18 & 9.85 & 1.13 & $3.56^{* *}$ \\
\hline ARM-248A $\times$ LTRR-341 & 35.78 & 0.52 & $4.71 * *$ & 9.74 & 2.14 & -0.15 \\
\hline ARM-248A $\times 3376 R$ & 38.13 & 0.49 & 0.08 & 11.64 & -0.60 & $1.31 * *$ \\
\hline ARM-248A $\times$ RHA-6D-1R & 39.48 & -0.40 & -0.46 & 14.36 & $2.79 *$ & -0.18 \\
\hline ARM-248A $\times$ R-298 & 38.65 & 1.51 & 0.90 & 10.42 & 1.43 & $2.22 * *$ \\
\hline ARM-248A $\times$ R-649 & 38.84 & $-0.03 *$ & -0.57 & 11.37 & 1.17 & 0.24 \\
\hline KBSH 1 & 37.37 & 1.12 & -0.39 & 11.62 & 0.23 & 0.00 \\
\hline PAC 1091 & 35.28 & 0.44 & $6.83 * *$ & 12.12 & 0.23 & -0.03 \\
\hline KBSH 44 & 35.70 & 1.94 & -0.38 & 13.08 & 1.03 & $1.31 * *$ \\
\hline SE of bi & & 0.84 & & & 0.57 & \\
\hline
\end{tabular}

Contd...

*: Significant at $5 \%$ level; **: Significant at $1 \%$ level 
All the parents except RES-834-1 and among the hybrids, three hybrids viz., CMS 17A $\times$ LTRR 341, ARM 243A $\times 3376 R$ and ARM $238 \mathrm{~A} \times 3376 \mathrm{R}$ recorded unit $(\mathrm{bi}=1)$ values and non-significant deviation from regression $\left(S^{2} \mathrm{di}=0\right)$ hence, they are considered as ideal and highly adaptable hybrids having average stability and expected to perform well in all the environments. These results are in conformity with the findings of Rukmini Devi et al., (2006) and Balu et al., (2007). Two hybrids CMS $234 \mathrm{~A} \times \mathrm{RHA}-6 \mathrm{D}-1 \mathrm{R}$ and CMS 852A $\times$ RHA$6 \mathrm{D}-1 \mathrm{R}$ recorded regression coefficient of more than one (bi>1) and hence are adaptable for favourable environments. For oil content, stability parameters revealed that five hybrids viz., CMS 89A $\times$ RES-834-1, CMS 852A $\times \mathrm{R}$ 649 ARM 238A $\times$ R 298, ARM 243A $\times$ RES834-1, and ARM 238A $\times$ RES-834-1 recorded higher mean value, unit regression $(\mathrm{bi}=1)$ values (Table 5) and hence, they are considered to be widely adaptable to different environments by possessing the average stability. Similar results were reported by Balu et al., (2007).

For oil yield per plant, performance of stability parameters revealed that all the parents except RES-834-1 possessed unit value of regression and minimum deviation from regression, hence they are widely adaptable with average stability. The testers LTRR 341 and R 649 recorded less than one of bi value hence is adaptable to poor environments with more than average stability. Among the hybrids, seven hybrids CMS 852A $\times$ RHA-6D-1R, CMS 89A $\times$ RES-834-1, ARM $243 \mathrm{~A} \times \mathrm{R} 298, \mathrm{ARM} 243 \mathrm{~A} \times 3376 \mathrm{R}, \mathrm{CMS} 89 \mathrm{~A}$ $\times$ RHA-6D-1R, ARM 238A $\times 3376 \mathrm{R}$ and ARM $243 \mathrm{~A} \times$ RHA-6D-1R recorded higher mean, unit regression $(\mathrm{bi}=1)$ values and hence, they are considered to be widely adaptable to different environments by possessing the average stability. While three hybrids ARM $238 \mathrm{~A} \times \mathrm{RES}-834-1, \mathrm{CMS} 234 \mathrm{~A} \times \mathrm{RHA}-6 \mathrm{D}-1 \mathrm{R}$ and ARM 248A $\times$ RHA-6D-1R recorded more than one of (bi>1) value hence are adaptable to favourable environments with less than average stability.

From the present study, it can be emphasized that among the parents CMS 17A, ARM 243A, 3376R and R-649 are stable for seed yield and yield components among the hybrids, three hybrids viz., CMS 17A × LTRR 341, ARM $243 \mathrm{~A} \times 3376 \mathrm{R}$ and $\mathrm{ARM} 238 \mathrm{~A} \times 3376 \mathrm{R}$ are identified as stable hybrids for seed yield per plant. In addition, the hybrid CMS 17A $\times$ LTRR 341 also possessed stability for days to $50 \%$ flowering and days to maturity, whereas the hybrids ARM 243A × 3376R and ARM 238A × 3376R showed stability for number of filled seeds per head and oil yield per plant. Two hybrids viz., CMS 234A $\times$ RHA-6D-1R and CMS 852A $\times$ RHA-6D-1R were identified and suitable for favourable environments. Further the hybrid CMS 852A $\times$ RHA-6D-1R showed average stability for plant height, head diameter, oil content and oil yield per plant. It could be concluded that the response of the genotypes to changing environments was not same for all the characters. None of the hybrid was found to possess a favourable combination of all stability parameters or ideal stability values for all the characters.

\section{Acknowledgements}

The first author is thankful to ICAR-Indian Institute of Oilseeds Research (Formerly DOR), Rajendranagar, Hyderabad-30 for providing germplasm and extending necessary facilities for carry out this research work.

\section{References}

Ahmed, S.B. and Abdalla, A.H. 2008. Genotype $\mathrm{x}$ environment interaction for yield and yield components in some sunflower (Helianthus annuus L.) hybrids. University of Khartoum Journal of American Sciences, 16 (1): 15-31

Balu, P. A., Sumathi, P., Ibrahim, S. M. and Kalaimagal, T. 2007. G $\times$ E interaction and stability analysis in sunflower (Helianthus annuus L.). Indian J. Genet. Plant Breed. 67(4): 388-391.

Breeze, E.L. 1969. The measurement and significance of genotype - environment 
interactions in grasses. Heredity 24:181200.

Cooper, M. and De-Lacy, I.H. 1994. Relationships among analytical methods used to study genotypic variations and genotype-by-environment interaction in plant breeding multi environment experiments. Theoretical Applied Genetics, 88: 561-572

Eberhart, S. A. and Russell, W. A. 1966. Stability parameters for comparing varieties. Crop Sci. 6: 36-40.

Halaswamy, B. H., Bhushana, H. O., Kulkarni, R. S., Shekar, G.C. and Seetharam, A. 2001. Analysis of three-way cross sunflower hybrids for yield and oil content over different environments. Crop Research 21(2): 204-207.

Kandil, A., Ibrahim, A. F., Marquarld, R. and Taha, R.R. 1990. Response of some quality traits of sunflower seeds and oil to different environments. J. agron. 164 (4): 22-30

Kang, M.S. and Gorman, D.P. 1989. Genotype $\mathrm{x}$ environment interaction in maize. Agron. J., 81 (4): 662-664

Laishram, J. M. and Singh, N. N. 1997. Phenotypic stability for qualitative characters in sunflower (Helianthus annuus L.) in Manipur. Indian J. Genet. Plant Breed. 57(2): 174-179.

Mahajan, R. C., Ghodke, M. K., Gir, S. N. and Jagtap, P. K. 2009. Stability analysis and heterosis behaviour in newly developed hybrids of sunflower (Helianthus annuus L.). J. Oilseeds Res., 26 (Special issue): 689-691.

Murali, M. R., Ranganatha, A. R. G., Hemanth Kumar, M., Rukmini Devi, K. and
Narendre, B. 2004. Adaptability of sunflower hybrids to crop growing seasons of scarce rainfall zone. $J$. Maharashtra Agric. Univ., 29(2): 138140.

Neelima, S., Ashok Kumar, K., Venkataramanamma, K. and Padmalatha, Y.2016. Genetic variability and genetic diversity in sunflower. Elec. J. Plant Breed., 7(3): 703-707

Prusti, A. M., Bajaj, R. K., Subbalakshmi, B., Ganapathy, M., Dev, V. S. and Mohanty, J. K. 1999. Stability analysis in sunflower. Environ. Ecol. 17(1): 67-69.

Rukmini Devi, K., Ranganatha, A. R. G. and Ganesh, M. 2006. Analysis of diverse hybrids for stability to seed yield and yield components in sunflower. Ann. Agric. Res. 27(2): 167-178.

Shankar Goud, I. and Sarala, B. S. 2004. Stability for seed and oil yield in promising sunflower, Helianthus annuus L. hybrids under irrigated conditions in Northern parts of Karnataka. J. Oilseeds Res., 21(2): 329-330.

Shrestha, S.P., Asch, F., Dusserre, J., Ramanantsoanirine, A., Brueck, H. 2012. Climate effects on yield components as affected by genotypic responses to variable environmental conditions in upland rice systems at different altitudes. Field Crops Res, 134:216-228.

Sobhan Babu, S. 2005. Heterosis and stability studies on Petiolaris and Argophyllus cytoplasmic based hybrids in sunflower (Helianthus annuus L.) Ph.D Thesis, Acharya N.G. Ranga Agricultural University, Hyderabad.

\section{How to cite this article:}

Satish Chandra, B., S. Sudheer Kumar and Ranganatha, A.R.G. 2018. Genotype $\times$ Environment Interaction and Stability analysis for Seed Yield and its Component Traits in Sunflower (Helianthus annuus L.). Int.J.Curr.Microbiol.App.Sci. 7(11): 170-182. doi: https://doi.org/10.20546/ijcmas.2018.711.021 